\title{
An Egyptian study for standardization of myocardial T1 mapping values on a 3 Tesla MRI machine
}

\author{
Ahmed Samir Ibrahim, Emad Hamid Abdel-Dayem, Hossam Mohsen Hassan \\ Radiology Department, Faculty of Medicine, Ain Shams University, Cairo, Egypt \\ Corresponding author: Hossam Mohsen Hassan,email: hosammohsen91@ gmail.com,Mobile: 01110636022
}

\begin{abstract}
Objective: of this study is to set standard values for the native T1 values in both normal and diseased myocardium on a 3 tesla MRI machine.

Methodology: this study was carried out in Misr Radiology Center. 31 patients were divided into normal group (control) including 10 patients and diseased group including 21 patients.

Result: The native T1 values on the healthy myocardium ranged from 1110 to $1300 \mathrm{msec}$, with ECV values ranging from 25 to $33 \%$ with variable elevations of the native T1 and ECV values according to the pathology affecting the myocardium. We concluded that the above values are the reference values for the $3 \mathrm{~T}$ MRI machine.
\end{abstract}

Keywords: Cardiovascular Magnetic Resonance (CMR), Extra-cellular Volume (ECV), Late Gadolinium Enhancement (LGE).

\section{INTRODUCTION}

In the heart, diffuse interstitial fibrosis plays an essential role in the development of a variety of cardiomyopathies and is associated with increased mortality. Previously, endomyocardial biopsy was the principle method used to diagnose myocardial fibrosis. Currently, T1 mapping is a novel and expanding application of cardiac MR imaging and has the potential to depict diffuse interstitial fibrosis in a variety of cardiac diseases ${ }^{(I)}$.

Rapid innovations in CMR now permit the routine acquisition of quantitative measures of myocardial and blood $\mathrm{T} 1$ which are key tissue characteristics. T1 quantification requires the acquisition of multiple images to derive the $\mathrm{T} 1$ recovery curve which is governed by the exponential time constant for MR longitudinal relaxation, T1. This parameter can be displayed as a pixelwise "T1 map" whereby an estimate of $\mathrm{T} 1$ is encoded in the intensity of each pixel. Its quantitative nature permits establishing normal $\mathrm{T} 1$ ranges, and $\mathrm{T} 1$ values can be assigned colors to simplify visual interpretation ${ }^{(2)}$.

Native T1-mapping as well as ECV mapping is currently being explored as a diagnostic tool for a wide range of cardiomyopathies. Native $\mathrm{T} 1$ changes are detectable in both acute and chronic MI. Elevated native $\mathrm{T} 1$ has been reported in a number of diseases with cardiac involvement: myocarditis, amyloidosis, lupus and decrease in native $\mathrm{T} 1$ have been associated with Anderson Fabry disease, and high iron content ${ }^{(3)}$.

In chronic MI, there is replacement of myocardial cells by scarring or fibrosis with an increase in extracellular collagen. Importantly, there is no edema, as this has resolved in the initial weeks after MI. Therefore, T1 values are higher than in normal myocardium, but not as high as in acute MI (4).

Native T1-mapping can display the typical non-ischemic patterns in acute myocarditis, similar to LGE imaging without the need for contrast agents. T1-mapping also detected additional areas of myocardial involvement and identified extra cases beyond T2W and LGE imaging ${ }^{(5)}$.

A relatively higher pre-contrast $\mathrm{T} 1$ value and $\mathrm{ECV}$, and lower post-contrast $\mathrm{T} 1$ value were found with $\mathrm{T} 1$ mapping in the myocardium of HCM patients, which suggested T1 mapping is better in the evaluation of myocardial fibrosis ${ }^{(6)}$.

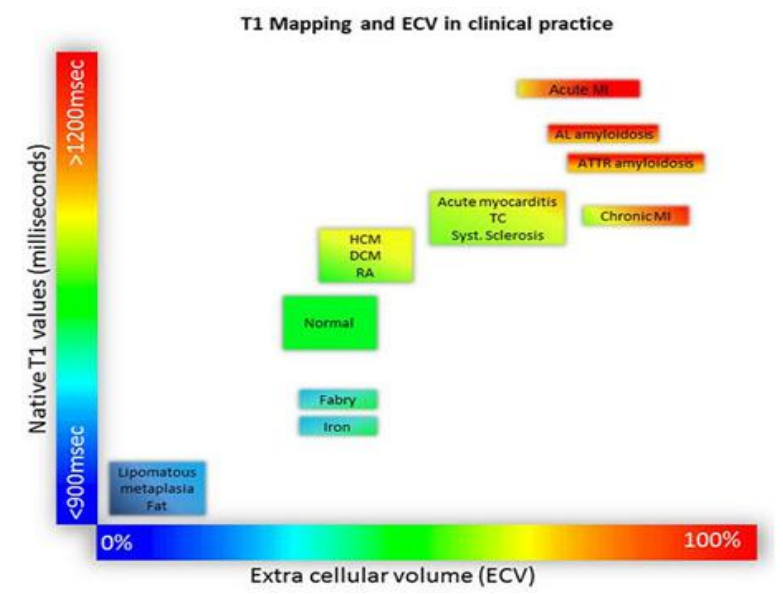

Figure (1): Tissue characterization using native T1 and extracellular volume fraction (ECV). Absolute values for native $\mathrm{T} 1$ depend greatly on field strength $(1.5 \mathrm{~T}$ or 3 $\mathrm{T}$ ), pulse sequence (MOLLI or ShMOLLI), scanner manufacturer and rules of measurements ${ }^{(7)}$.

T1 mapping is hypothesized to contribute to the characterization of cardiac masses based on the spectrum of $\mathrm{T} 1$ relaxation times in tissue consisting of fat, calcium, melanin, blood and simple fluid. Thrombi and myxomas showed 
intermediate and relatively long $\mathrm{T} 1$ times, respectively ${ }^{(8)}$.

Broadly, T1 mapping sequences have three parts: (1) the T1 magnetization preparation pulse, (2) a single image acquisition (readout) after a variable delay, and (3) variable repetitions of (1) and (2) to sample the longitudinal magnetization recovery curve after the magnetization preparation. Raw images are then reconstructed by postprocessing into a single $\mathrm{T} 1$ map using a theoretical model of the expected signal intensity ${ }^{(9)}$.

A T1 map is a two-dimensional (usually brightly colored) slice image where each image pixel displays the $\mathrm{T} 1$ relaxation time (ms) using a color look-up table to facilitate visual assessment ${ }^{(10)}$.

Pixel-wise T1-mapping first appeared on the scene with the introduction of the MOLLI imaging strategy, which propelled the use of T1mapping in CMR and inspired many new methods. MOLLI is widely used today with some protocol optimization and other adaptations ${ }^{(11)}$.

Table (1): Summary of pros and cons of various T1 mapping methods ${ }^{(12)}$.

\begin{tabular}{|c|c|c|c|}
\hline $\begin{array}{c}\text { Point of } \\
\text { comparison }\end{array}$ & MOLLI & SHMOLLI & SASHA \\
\hline $\begin{array}{c}\text { Short } \\
\text { breath hold }\end{array}$ & Poor & Fair & Fair \\
\hline $\begin{array}{c}\text { HR } \\
\text { insensitivity }\end{array}$ & Poor & Fair & Fair \\
\hline $\begin{array}{c}\text { Absolute } \\
\text { accuracy }\end{array}$ & Poor & Poor & Good \\
\hline $\begin{array}{c}\text { Signal to } \\
\text { noise ratio }\end{array}$ & Good & Good & Poor \\
\hline
\end{tabular}

\section{AIM OF THE WORK}

We aim to determine the standard native T1 values specific to the Siemens 3 Tesla machine in both normal and diseased myocardia in an Egyptian population.

\section{PATIENTS AND METHODS}

\section{Patients:}

During a period of 12 months' duration from May 2017, thirty-one patients were enrolled in the study. Patients with clinical symptoms suggestive of underlying cardiac condition (e.g., dyspnea, chest pain or easy fatigability); whether suspected by another imaging modality (e.g., echocardiographs) or not.
Inclusion Criteria: All Patients asked to do Cardiac MRI; normal and diseased. Both sexes will be included. Adults and children.

Exclusion Criteria: Contraindications to MRI such as claustrophobia, pacemakers, cochlear implants, etc. Patients with degenerative neurological disorders that makes them unstable during the test. Patients with bad general condition needing life support.

MRI imaging: The study was performed with a 3-T MR system (Magnetom TIM TRIO, Siemens, Erlangen, Germany).

T1 mapping study: In addition to the routine CMR sequences; Modified Look-Locker. Inversion recovery pulse sequence is used. For T1 mapping, data were acquired in basal, midventricular, and apical SAX planes before and 10 - minutes after administration of $0.3 \mathrm{mmol} / \mathrm{kg}$ i.v. gadobutrol (Gadovist ${ }^{\circledR}$, Bayer Healthcare Germany). Data were obtained in end-diastole using a cardiac gated, SSFP-based modified looklocker inversion recovery (MOLLI) technique.

MRI data analysis: Multiple parameters were measured: End-diastolic and end-systolic left ventricle volumes, ejection fraction, stroke volume and left ventricle mass for assessment of the ventricular function. Assessment of any anatomical variants or pathologies within the cardiac chambers e.g., masses or thrombi. Wall motion of the left ventricle is assessed at each myocardial segment: Normal, hypokinetic, akinetic, or dyskinetic. Detection if any late gadolinium enhancement was present throughout any of the 17 heart segments based on the 17-segment model recommended by the AHA (American Heart Association). Pixel wise illustration of the absolute $\mathrm{T} 1$ relaxation times on a map was done, color-coded maps were obtained. Then the ROI was placed over the 17 heart segments in both pre and post contrast images according to the AHA; that are 6 basal segments, 6 mid ventricular segments and 4 apical segments in addition to the blood itself in order to obtain the pre and the postcontrast $\mathrm{T} 1$ values in each of these segments. Extra-Cellular Volume is then calculated according to specific equation:

$\mathrm{ECV}=(1-$ hematocrit $) \mathrm{x}$ 

$\frac{1}{\text { postcontrast } T 1 \text { myocardium }}-\frac{1}{\text { Native } T 1 \text { myocardium }}$ $\overline{\text { Post contrast T1 Myocardium }}-\frac{1}{\text { Native } T 1 \text { blood }}$
IBM SPSS statistics (V. 25.0, IBM Corp., USA, 20172018) was used for data analysis. Date were expressed as mean \pm SD for quantitative parametric measures in addition to both number and percentage for categorized data. Crosstabulation was used to describe the categorized data.

\section{Statistical analysis}

\section{RESULTS}

The tables below show that most of our study population were males representing about $83.8 \%$ as well as the sex distribution among the control and the diseased.

Table (2): Shows the mean age of the control group and the standard deviation as well as the sex distribution among the group.

\begin{tabular}{|c|c|c|}
\hline & & Control group \\
\hline & & No. $=\mathbf{1 0}$ \\
\hline Age & $\begin{array}{l}\text { Mean } \pm \text { SD } \\
\text { Range }\end{array}$ & $\begin{array}{c}45.20 \pm 18.86 \\
12-78\end{array}$ \\
\hline Sex & $\begin{array}{l}\text { Female } \\
\text { Male }\end{array}$ & $\begin{array}{l}2(20.0 \%) \\
8(80.0 \%)\end{array}$ \\
\hline
\end{tabular}

Table (3): Shows the mean age of the diseased group and the standard deviation as well as the sex distribution among the group.

\begin{tabular}{|c|c|c|}
\hline & Patients group \\
\hline & & No. $=21$ \\
\hline Age & $\begin{array}{l}\text { Mean } \pm \text { SD } \\
\text { Range }\end{array}$ & $\begin{array}{c}52.67 \pm 15.70 \\
28-77\end{array}$ \\
\hline Sex & $\begin{array}{l}\text { Female } \\
\text { Male }\end{array}$ & $\begin{array}{c}3(14.3 \%) \\
18(85.7 \%)\end{array}$ \\
\hline
\end{tabular}

So among 10 patients who underwent cardiac MRI and were found to have healthy myocardium; we calculated the mean native $\mathrm{T} 1$ value and standard deviation from the mean value.

Table (4): Shows the mean native, postcontrast T1 values as well as ECV values in the control group.

\begin{tabular}{|l|c|c|c|}
\hline \multirow{2}{*}{} & Control group & Control group & Control group \\
\cline { 2 - 4 } & Mean \pm SD & Mean \pm SD & Mean \pm SD \\
\cline { 2 - 4 } & Native T1 & Post Contrast T1 & ECV \\
\hline Basal & & & \\
\hline Ant & $1198.80 \pm 39.22$ & $527.10 \pm 80.21$ & $28.80 \pm 6.66$ \\
\hline IS & $1224.40 \pm 76.58$ & $519.30 \pm 76.78$ & $30.30 \pm 7.75$ \\
\hline Inf & $1221.30 \pm 63.96$ & $517.40 \pm 90.64$ & $30.50 \pm 8.28$ \\
\hline IL & $1243.40 \pm 65.62$ & $491.80 \pm 90.85$ & $33.70 \pm 7.80$ \\
\hline AL & $1192.30 \pm 40.69$ & $505.70 \pm 81.31$ & $31.50 \pm 8.03$ \\
\hline Mid Ventricle & $1215.20 \pm 40.72$ & $528.60 \pm 74.77$ & $29.00 \pm 7.10$ \\
\hline Ant. & & & $27.80 \pm 5.63$ \\
\hline AS & $1190.50 \pm 73.12$ & $535.20 \pm 79.38$ & $29.00 \pm 6.86$ \\
\hline IS & $1210.60 \pm 43.83$ & $528.90 \pm 79.82$ & $28.50 \pm 5.48$ \\
\hline Inf. & $1210.80 \pm 42.15$ & $532.10 \pm 82.27$ & $28.60 \pm 6.26$ \\
\hline IL & $1220.80 \pm 25.05$ & $533.00 \pm 92.41$ & $28.00 \pm 6.57$ \\
\hline AL & $1161.90 \pm 52.38$ & $526.40 \pm 81.13$ & \\
\hline Apex & $1209.50 \pm 40.90$ & $538.80 \pm 78.76$ & $27.90 \pm 6.44$ \\
\hline Ant & & & $523.50 \pm 74.39$ \\
\hline
\end{tabular}


An Egyptian study for standardization of myocardial T1 mapping values ....

\begin{tabular}{|l|l|l|c|}
\hline Sept & $1189.70 \pm 51.20$ & $527.60 \pm 74.05$ & $28.90 \pm 7.88$ \\
\hline Inf. & $1229.40 \pm 55.22$ & $550.40 \pm 57.89$ & $27.70 \pm 8.15$ \\
\hline Lat. & $1177.70 \pm 55.49$ & $524.40 \pm 79.90$ & $29.20 \pm 8.02$ \\
\hline
\end{tabular}

From the above tables; we can say that a native $\mathrm{T} 1$ ranging from $1110 \mathrm{msec}$ to $1300 \mathrm{msec}$ usually denotes a healthy myocardium with no scarring, fibrosis or infiltration on the 3 tesla MRI machine.

And that healthy myocardium usually shows ECV values ranging from $25 \%$ to $35 \%$.

Then, we classify our diseased patients according to the myocardial pathology as described in the table below:

Table (5): Describes the percentage of the diseased patients according to the myocardial pathology in the diseased group.

\begin{tabular}{|l|c|c|}
\hline Type of diseased & No. & \% \\
\hline Amyloidosis & 1 & $4.8 \%$ \\
\hline HOCM & 4 & $19 \%$ \\
\hline Infarction & 9 & $42.9 \%$ \\
\hline Masses & 6 & $28.6 \%$ \\
\hline Myocarditis & 1 & $4.8 \%$ \\
\hline Total & 21 & $100.0 \%$ \\
\hline
\end{tabular}

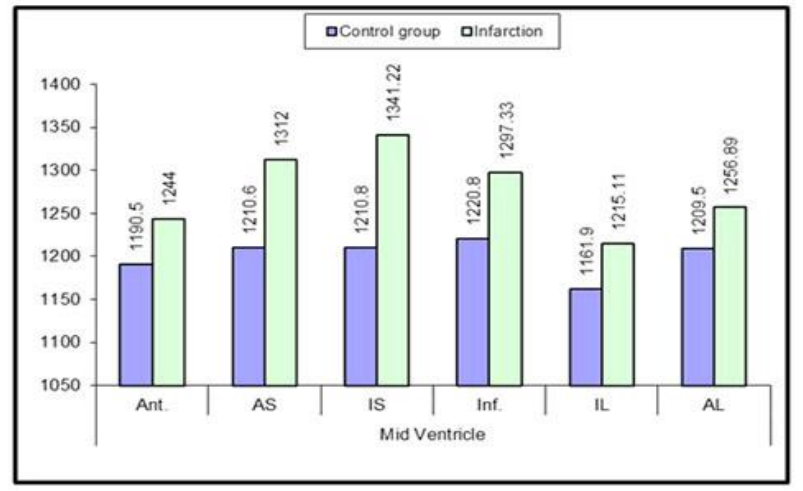

Figure (2): Shows elevated native T1 values in the patient's group compared to the healthy population.

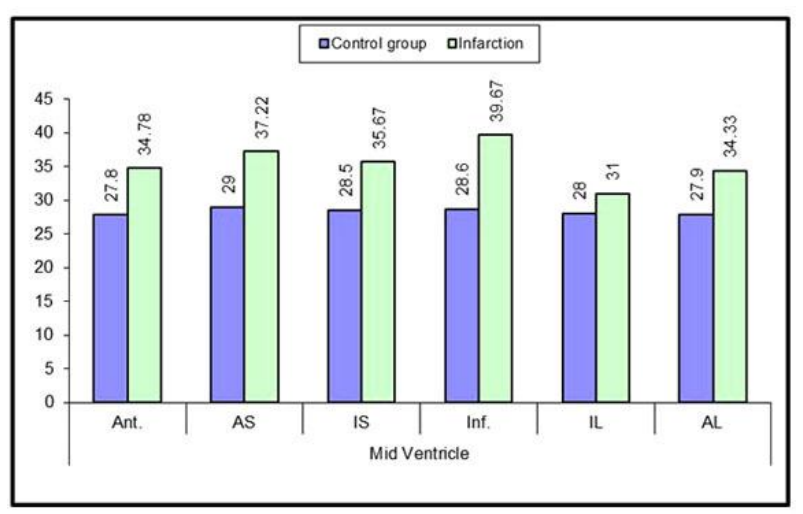

Figure (3): Elevated ECV values in those with myocardial infarctions compared to the control group in the mid myocardial segments.

The previous data can be made clearer by the following example of a 55 years old male patient with Ischemic heart disease and past history of PCI twice.

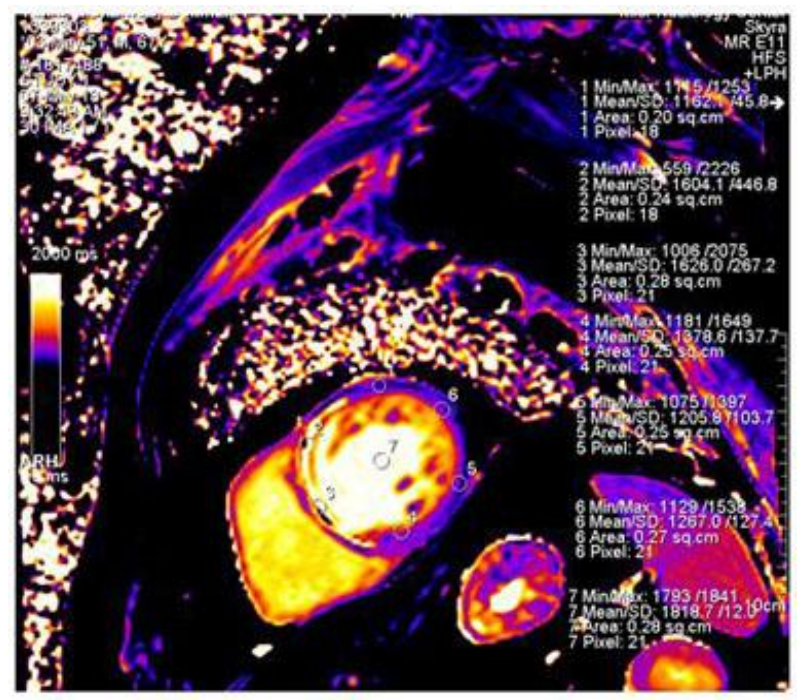

Figure (4): Delayed Post-contrast image in the mid ventricular region showing post contrast enhancement in the anterolateral, infero-septal and inferior mid ventricular walls.

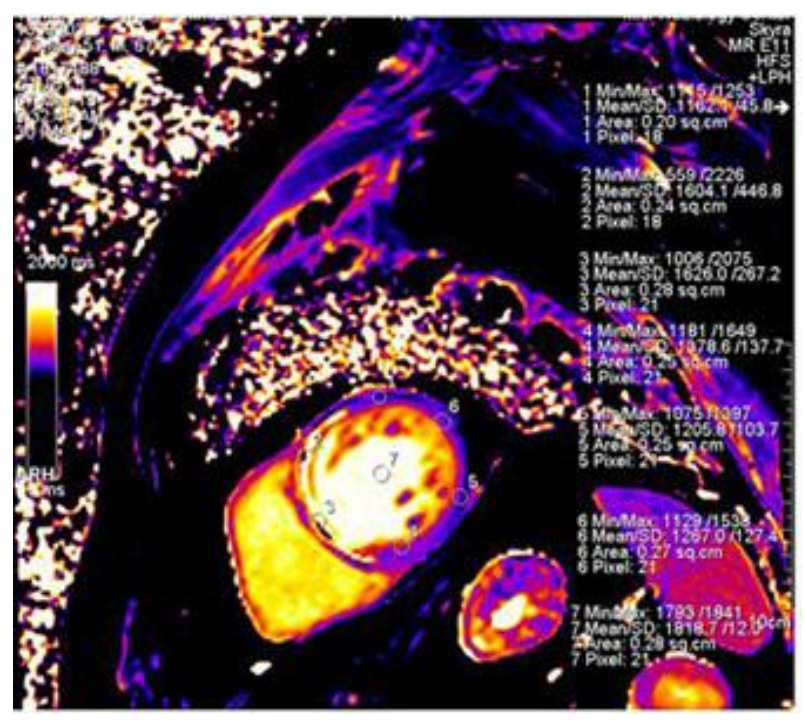


Figure (5): T1 colour coded images in mid ventricular region showing elevated native $\mathrm{T} 1$ values in the anterolateral, infero-septal and inferior mid ventricular segments.

Table (6): Native T1 and ECV values in a patient with infarctions involving the anterolateral, inferoseptall and inferior mid ventricular segments.

\begin{tabular}{|l|c|c|}
\hline \multicolumn{1}{|c|}{ Myocardial Segment } & $\begin{array}{c}\text { Native T1 } \\
\text { values }\end{array}$ & $\begin{array}{c}\text { ECV } \\
\text { values }\end{array}$ \\
\hline 1-Anterior & $1=1162$ & $1=28$ \\
2-Anteroseptal & $2=1604$ & $2=47$ \\
\hline 3-Inferoseptal & $3=1626$ & $3=45$ \\
4-Inferior & $4=1379$ & $4=36$ \\
\hline 5-Inferolateral & $5=1206$ & $5=33$ \\
6-Anterolateral & $6=1267$ & $6=33$ \\
\hline
\end{tabular}

Also, significantly higher native $\mathrm{T} 1$ and ECV values were reported in patients with hypertrophic cardiomyopathies entailing scarring or fibrosis of one or more of the myocardial segments.

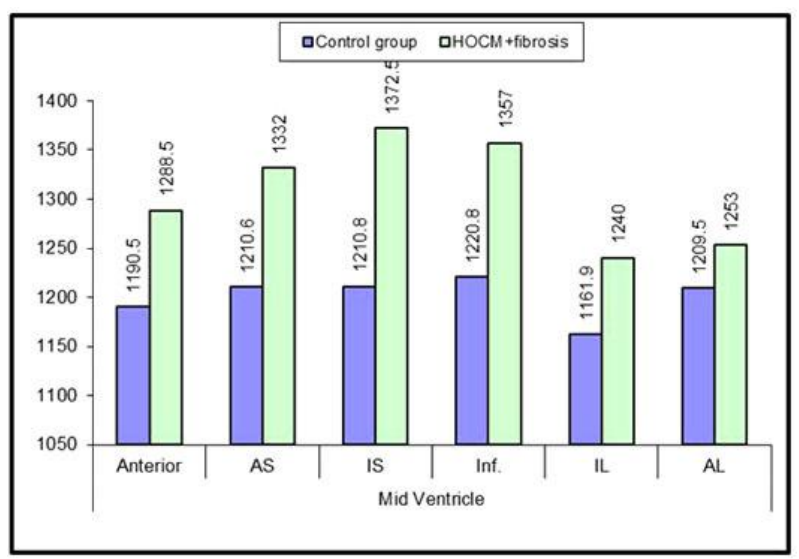

Figure (6): Significantly elevated $\mathrm{T} 1$ values in those with myocardial scarring duo to HOCM compared to the normal population.

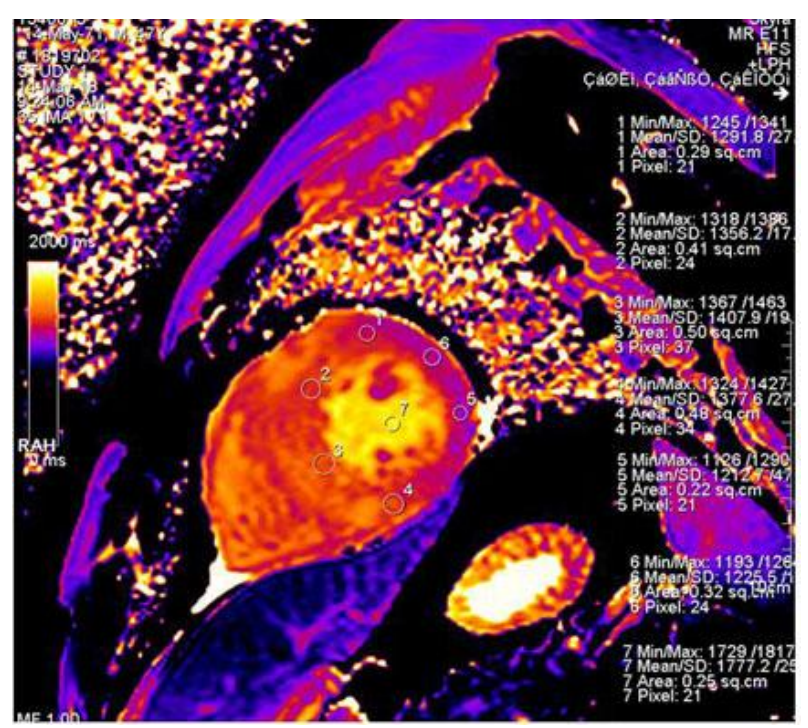

Figure (7): Color coded map representing the elevated native $\mathrm{T} 1$ values in the scarred myocardial segments in a patient of HCM.

Our study included one patient with cardiac amyloidosis and one patient with chronic myocarditis Elevations of the native T1 values and ECV values were noted all over most of the myocardial segments compared to the control group.

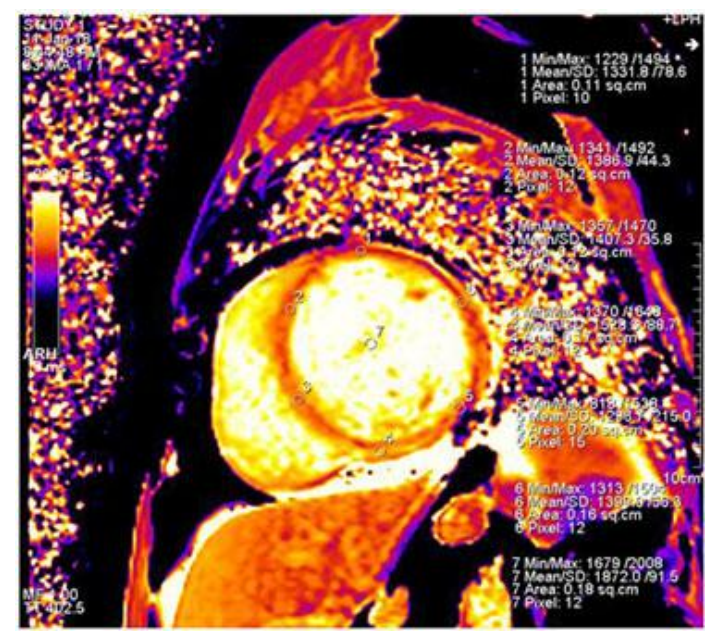

Figure (8): Color coded T1 image of the mid ventricular myocardium showing diffuse elevation of the native $\mathrm{T} 1$ values in patent with chronic myocarditis. 


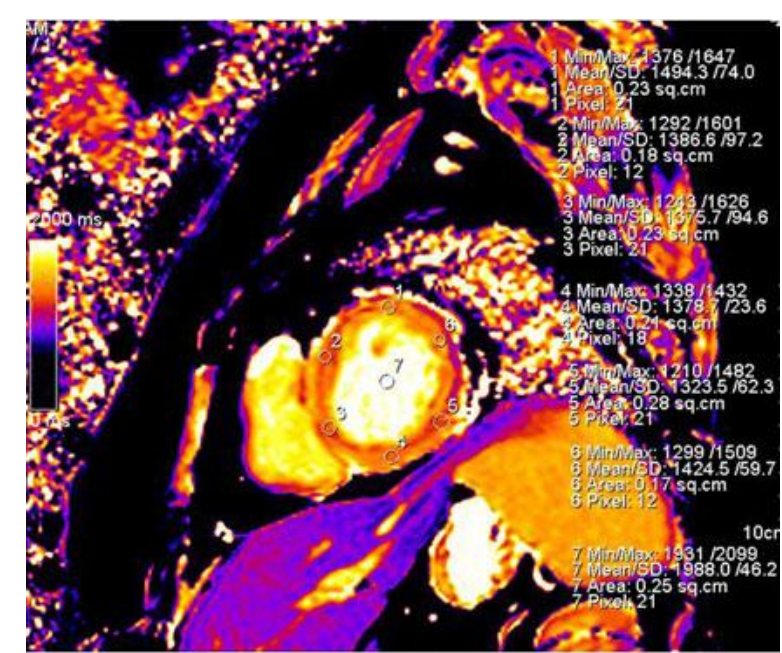

Figure (9): Color coded T1 image of the mid ventricular myocardium showing diffuse elevation of the native $\mathrm{T} 1$ values in patent with cardiac amyloidosis.

Our study included 6 patients with cardiac masses representing $28.6 \%$ of the patients group.

These masses included cardiac thrombi and neoplastic masses.

\section{i- Cardiac thrombi:}

One of the two patients that were referred to us with cardiac thrombi detected on echocardiography was not injected contrast agent duo to the elevated kidney functions. So, post contrast $\mathrm{T} 1$ value was not obtained.

The mean native $\mathrm{T} 1$ value of the cardiac thrombus was $1065.5 \mathrm{msec}$.

\section{ii- Cardiac neoplastic masses:}

Three patients with neoplastic masses or thrombi were referred to us. They showed variable native and post contrast $\mathrm{T} 1$ values.

Mean native $\mathrm{T} 1$ value $=1035 \mathrm{msec}$.

Mean post contrast T1 value $=541.6 \mathrm{msec}$.

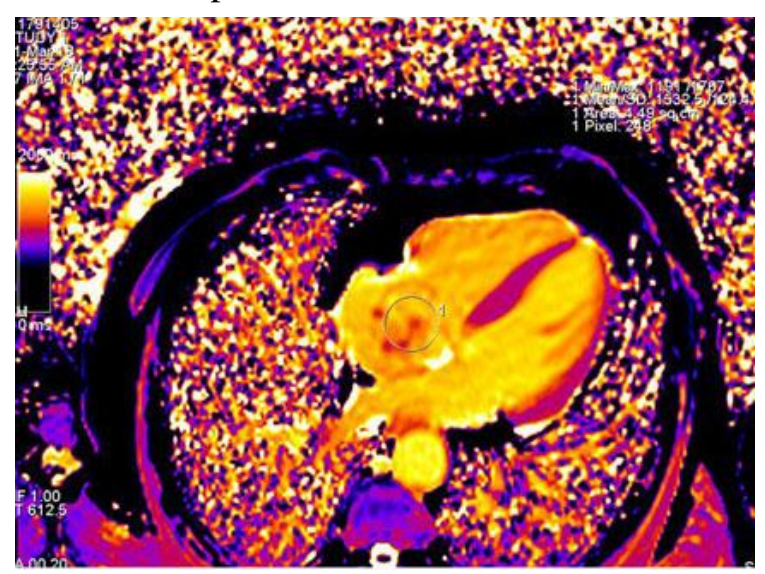

Figure (10): Color coded T1 image showing elevated native $\mathrm{T} 1$ value of the lesion.

\section{DISCUSSION}

Cardiovascular magnetic resonance (CMR) provides techniques for non-invasive myocardial tissue characterization. T1 mapping of the left ventricular myocardium, i.e. quantification of the myocardial $\mathrm{T} 1$ relaxation time, as well as the T1derived extracellular volume fraction have been demonstrated to add valuable information ${ }^{(13)}$.

The differences in acquisition schemes have a direct effect on the range of normal and abnormal T1 with a given technique, which means that absolute $\mathrm{T} 1$ values can only be directly compared when they were obtained with the same acquisition scheme at the same field strength using the same post-processing methods. Thus, reports on $\mathrm{T} 1$ values should always include the T1 mapping technique that was used and the site-specific normal range for $\mathrm{T} 1^{(14)}$.

Our main finding on the normal population were: $\mathrm{T} 1$ mapping achieved a high degree of diagnostic image quality. Observer dependency of $\mathrm{T} 1$ relaxation time quantification was very low. Mean values of myocardial T1 relaxation times are presented per segment and per slice and can be used as reference values in the next CMR examination entailing the use of $\mathrm{T} 1$ mapping sequences. An inter-subject variation of the T1 values is present and this can be a limiting factor to establishment of standard cut-off values. The myocardial $\mathrm{T} 1$ relaxation times reported here can be regarded as reference values specific only for this cohort, time point, mapping technique, type and dosage of contrast media. Further comparisons with other published results are difficult unless an identical study design is used.

A study performed on 2016 by Weingartner et al., on 20 healthy subjects on a 3 T MRI machine concluded that the mean native $\mathrm{T} 1$ value using MOLLI technique was $1182.6+/-35.8$ msec., however the post contrast $\mathrm{T} 1$ value was $541.1+/-33.8$ msec and ECV values $27.5+/-3.1 \%{ }^{(15)}$.

Among the healthy population of our study; our mean precontrast $\mathrm{T} 1$ value is $=1209 \mathrm{msec}$ among all the myocardial segments, while the mean postcontrast $\mathrm{T} 1$ value $=525.6 \mathrm{msec}$. and the mean ECV was $29.3 \%$.

Dall'Armellina et al. conducted a study published in 2012 aiming to define the native T1 
values in cases of myocardial infarctions. They reported a mean precontrast T1 value of $1257 \mathrm{msec}$. for infarcted segments compared to $1196 \mathrm{msec}$ for normal unaffected segments at 3 T MRI ${ }^{(16)}$.

In our study; we reported a mean precontrast T1 value of $1369 \mathrm{msec}$ for the infarcted myocardial segments compared to 1209 for the normal unaffected segments.

Liu et al. stated that in patients with HOCM; the pre-contrast T1 value was $1217.3 \pm$ 97.4 msec. with an ECV $25.7 \pm 3.6 \%$.

In our study; the findings are higher than the above study; as we reported a mean native T1 in HCM patients of $1339 \mathrm{msec}$ and mean ECV of 38\%; this is duo to the fact that our study was conducted over a $3 \mathrm{~T}$ MRI machine which resulted in higher T1 values.

In a study performed by $\boldsymbol{L}$ in et $\boldsymbol{a l}$. in 2018 on 82 patients with cardiac amyloidosis and 20 healthy subjects on a 3T scanner; the patients demonstrated an increase in native $\mathrm{T} 1 \mathrm{of} 1438 \pm 120 \mathrm{~ms}$ vs. $1283 \pm 46 \mathrm{~ms}$ for the control group and ECV of $43.9 \pm 10.9 \%$ vs $27.0 \pm 1.7 \%$ for the healthy controls ${ }^{(17)}$.

In our study; we got similar measurements of the native T1 and the ECV values among the patient of myocarditis reaching $1384.5 \mathrm{msec}$. and $44 \%$ respectively.

In patients with myocarditis, we found in our study that segments with LGE had significantly higher T1 and ECV values, and patient segments without LGE had less elevated T1 values but still above the normal range, suggesting that areas without apparent LGE were also involved.

In a study done by Casper et al. in 2017 on 19 patients with cardiac masses; both myxomas and neoplastic masses showed relatively long native T1 values measuring $1316 \pm 71 \mathrm{msec}$ and $1333 \pm 101$ msec respectively ${ }^{(18)}$.

Our results in both benign (myxoma) and malignant cardiac masses are much less than the above-mentioned values as they showed $\mathrm{T} 1$ values of $253 \mathrm{msec}$ and $1035 \mathrm{msec}$ respectively; this is mostly duo to small number of the cases or in-appropriate defining of the lesions on the color-coded maps.

\section{CONCLUSION}

Tissue characterization by native $\mathrm{T} 1$ mapping may serve as an important source of diagnostic, therapeutic and prognostic decision making in various cardiac diseases. An advantage of a non-invasive method for the assessment of fibrosis is the potential to follow changes in the myocardium over time as in patients with cardiomyopathies Patients with poor renal function (or on dialysis) precluding gadolinium-based contrast injection may benefit from using native $\mathrm{T} 1$ mapping instead of LGE imaging. Clinically, several studies have shown that T1 mapping with ECV is particularly useful in the assessment of cardiac diseases with diffuse fibrosis. Furthermore, T1 mapping with ECV might be helpful as an adjunct in cases with ambiguous LGE. Beyond differential diagnosis of cardiomyopathies, tissue characterization with $\mathrm{T} 1$ mapping can be very useful in differentiating between pericardial fat vs. LGE as well as in tissue characterization of various cardiac tumors.

Although tissue characterization with native $\mathrm{T} 1$ and ECV has been shown to have incremental diagnostic benefit even in very early disease stages (e.g. diffuse fibrosis not detectable by LGE), there is an overlap between different cardiomyopathies and some overlap with normal $\mathrm{T} 1$ values. Like all medical parameters, abnormalities in native T1 and ECV need to be interpreted within their clinical context and pre-test probabilities and in conjunction with established CMR techniques such as LGE. Elevations and reductions of T1 and ECV are not specific and can be caused by various disease processes. In some instances, these processes can even cancel each other out (e.g., pseudonormalization in AndersonFabry disease when replacement fibrosis exceeds the fatty-related $\mathrm{T} 1$ decrease).

\section{REFERENCES}

1. Jeremy RB, Stefan LZ, Ihab RK et al. (2014): Myocardial T1 Mapping: Techniques and Potential Applications RadioGraphics, 34:377-395

2. Moon JC, Messroghli DR, Kellman $P$ et al. (2013): Myocardial T1 mapping and extracellular volume quantification: a Society for Cardiovascular Magnetic Resonance (SCMR) and CMR Working Group of the European Society of Cardiology consensus statement. Journal of Cardiovascular Magnetic Resonance, 15:92.

3 Kellman P and Hansen MS (2014): T1-mapping in the heart: accuracy and precision. Journal of Cardiovascular Magnetic Resonance, 16:2. 
4 H-Ici DO, Jeuthe S, Al-Wakeel $\mathrm{N}$ et al. (2014): $\mathrm{T} 1$ mapping in ischaemic heart disease. Eur Heart J Cardiovasc Imag., 15:597-602.

5 Ferreira VM, Piechnik SK, Dall'Armellina E et al. (2014): Native T1-mapping detects the location, extent and patterns of acute myocarditis without the need for gadolinium contrast agents. Journal of Cardiovascular Magnetic Resonance, 16:36.

6 Liu Y, Qi S, Wang Z et al. (2016): T1 mapping for characterization of myocardial fibrosis in hypertrophic cardiomyopathy. Journal of Cardiovascular Magnetic Resonance, 18(Suppl 1):320.

7 Haaf P, Garg P, Messroghli DR et al. (2016): Cardiac T1 Mapping and Extracellular volume (ECV) in clinical practice: a comprehensive review. Journal of Cardiovascular Magnetic Resonance, 308:18-89.

8 Saba SG, Bandettini PW, Shanbhag SM et al. (2015): Characterization of cardiac masses with T1mapping. Journal of Cardiovascular Magnetic Resonance, 17(Suppl 1):32.

9 Higgins DM and Moon JC (2014): Review of T1 mapping methods: comparative effectiveness including reproducibility issues. Curr Cardiovasc Imaging Rep., 7:9252.

10 Everett RJ, Stirrat CG, Semple SIR et al. (2016): Assessment of myocardial fibrosis with T1 mapping MRI. Clin Radiol., 71:768-778

11 Messroghli DR, Radjenovic A, Kozerke S et al. (2004): Modified Look-Locker inversion recovery (MOLLI) for highresolution T1 mapping of the heart. Magn Reson Med., 52:141-6.

12 Kellman $P$ and Hansen MS (2014): T1mapping in the heart: accuracy and precision.
Journal of Cardiovascular Magnetic Resonance, 16:2.

13 Knobelsdorff-Brenkenhoff F, Prothmann M, Dieringer MA et al. (2013): Myocardial T1 and T2 mapping at $3 \mathrm{~T}$ : reference values, influencing factors and implications. Journal of Cardiovascular Magnetic Resonance, 15:53.

14 Moon JC, Messroghli DR, Kellman P et al. (2013): Myocardial T1 mapping and extracellular volume quantification: a Society for Cardiovascular Magnetic Resonance (SCMR) and CMR Working Group of the European Society of Cardiology consensus statement. Journal of Cardiovascular Magnetic Resonance, 15:92.

15 Kawel N, Nacif M, Zavodni A et al. (2012): T1 mapping of the myocardium: Intraindividual assessment of the effect of field strength, cardiac cycle and variation by myocardial region. J Cardiovasc Magn Reson., $14: 27$.

16 Weingartner S, Mebner NM, Budjan J et al. (2016): Myocardial $T_{1}$-mapping at $3 T$ using saturation-recovery: reference values, precision and comparison with MOLLI.Journal of cardiovascular Magnetic Resonance, 18: 84.

17 Dall'Armellina E, Piechnik SK, Ferreira VM et al. (2012): Cardiovascular magnetic resonance by non contrast T1- mapping allows assessment of severity of injury in acute myocardial infarction. J Cardiovasc Magn Reson., 14:15.

18 Caspar T, Ghannudi S, Ohana $M$ et al. (2017): Magnetic resonance evaluation of cardiac thrombi and masses by $\mathrm{T} 1$ and $\mathrm{T} 2$ mapping: an observational study Int $\mathrm{J}$ Cardiovasc Imaging, 33:551-559 